\title{
On the Origin of Mesoscopic Inhomogeneity of Conducting Polymers
}

\author{
Kevin O'Neil, Bryan Shaw, and Oleg A. Semenikhin* \\ Department of Chemistry, \\ The University of Western Ontario, \\ London, Ontario, N6A 5B7, Canada.
}

\section{Supporting Information}

The effect of the molar volume on the polymer nucleation according to the Kelvin model

The Kelvin equation gives the value of the critical radius $\boldsymbol{r}^{*}$ in the simplest case of the socalled capillarity approximation for clusters comprising sufficiently large number of molecules: ${ }^{1,2}$

$$
r^{*}=\frac{2 \cdot \sigma \cdot V_{M}}{R_{g a s} T \cdot \ln \left(\frac{c}{c_{s}}\right)}
$$

where $r^{*}$ is the critical radius of a cluster; 
c is the concentration of a nucleating species;

$c_{s} \quad$ is its saturated concentration;

$\boldsymbol{\sigma}$ is the surface tension at the polymer/solution interface;

$V_{M} \quad$ is the molar volume of the polymer;

$\boldsymbol{R}_{\text {gas }} \quad$ is the gas constant;

$\boldsymbol{T} \quad$ is the absolute temperature;

$r^{*} \quad$ is the critical radius of a nucleus;

$\frac{c}{c_{s}}=\zeta$ is the supersaturation ratio.

In addition to solubility, the molecular weight $(\mathrm{Mw})$ of a polymer fraction may affect also other parameters found in Eq. (S1), specifically, the polymer molar volume. At a first glance, the molar volume should increase with the molecular weight thus increasing the resulting critical radius and favoring a larger critical grain size. However, this apparent contradiction is easily resolved if we remember that, in the case of nucleation of molecules with different $\mathrm{Mw}$, the nuclei of the same size are comprised by a different number of molecules, and the survival of a nucleus will be determined not by its radius per se, but rather by the number of molecules required to form a nucleus of this size. Evidently, a critical nucleus that comprises a smaller number of molecules is more likely to be formed than a nucleus containing a larger number of molecules.

In order to better demonstrate this reasoning, the Kelvin equation (Eq. S1) can be re-written as (see Appendix for details):

$$
n^{*}=\frac{\pi}{6} \cdot \frac{(4 \sigma)^{3} \cdot V_{M}^{2}}{\left[R_{g a s} T \cdot \ln (\zeta)\right]^{3}}
$$


where $n^{*} \quad$ is the number of molecules of given Mw in a critical nucleus;

$V_{M} \quad$ is the molar volume of the polymer;

$$
V_{M}=N_{A} \cdot V_{0} ; V_{0}=\frac{V_{n u c l}}{n}
$$

where $\boldsymbol{V}_{\text {nucl }}$ is the volume of a single nucleus made of n polymer molecules;

$N_{A} \quad$ is Avogadro's number.

Equation (S2) allows one to see more clearly the predominant effect of supersaturation on the polymer nucleation from solutions containing a mixture of polymer molecules with different molecular weights. It is worth noting that it is assumed here that the volume of a polymer nucleus consisting of $\boldsymbol{n}$ polymer molecules is obtained by simple addition of the volumes occupied by individual molecules (a so-called liquid droplet model). However, longer chains can be expected to fold more efficiently upon forming a solid phase, thus resulting in a more densely packed and more crystalline structure. ${ }^{3,4}$ Therefore, one could expect that in fact the volume of the polymer nuclei formed by polymer molecules with higher $\mathrm{Mw}$ will be less than the value calculated using Eq. (S3). This should make the effect of supersaturation even more pronounced than predicted by Eq. (S2). 


\section{APPENDIX}

Following the paper of F.Q. Yu, ${ }^{5}$ let us use the liquid droplet model to find the Gibbs free energy for the addition of one polymer molecule to a cluster of $n-1$ polymer molecules of the same molecular weight:

$$
\Delta G_{n-1, n}=-k T \cdot \ln (\zeta)+\sigma \cdot\left(A_{n}-A_{n-1}\right)
$$

Here $\zeta=\frac{c}{c_{s}}$ is the supersaturation ratio;

$\sigma \quad$ is the surface tension at the polymer/solution interface;

$\boldsymbol{A}_{\boldsymbol{n}} \quad$ is the surface area of the polymer cluster containing n polymer molecules;

$\boldsymbol{A}_{n-1} \quad$ is the surface area of the polymer cluster containing n-1 polymer molecules;

$\boldsymbol{k} \quad$ is the Boltzmann constant;

$\boldsymbol{T} \quad$ is the absolute temperature.

Since the volume $\boldsymbol{V}_{\boldsymbol{n}}$ of the cluster containing $\boldsymbol{n}$ polymer molecules in terms of the liquid droplet model is equal to

$$
V_{n}=n \cdot V_{0}
$$

where $V_{\boldsymbol{0}}$ is the volume taken by one polymer molecule in the cluster,

we find the cluster radius $\boldsymbol{r}_{\boldsymbol{n}}$,

$$
r_{n}=\left(\frac{3 n V_{0}}{4 \pi}\right)^{\frac{1}{3}}
$$


Therefore,

$$
\begin{aligned}
& A_{n}-A_{n-1}=4 \pi \cdot\left(r_{n}^{2}-r_{n-1}^{2}\right)= \\
& =4 \pi \cdot\left(\frac{3 V_{0}}{4 \pi}\right)^{\frac{2}{3}} \cdot\left[n^{2 / 3}-(n-1)^{2 / 3}\right]= \\
& =(4 \pi)^{1 / 3} \cdot\left(3 V_{0}\right)^{2 / 3} \cdot n^{2 / 3} \cdot\left[1-\left(1-\frac{1}{n}\right)^{2 / 3}\right] \quad \text { when } n>>1 \\
& A_{n}-A_{n-1}=(4 \pi)^{1 / 3}\left(3 V_{0}\right)^{2 / 3} \cdot \frac{2}{3} n^{-1 / 3}
\end{aligned}
$$

For a critical nucleus,

$$
\Delta G_{n-1, n}=0
$$

and

$$
\begin{aligned}
& k T \cdot \ln (\zeta)=\sigma \cdot\left(A_{n}-A_{n-1}\right)= \\
& =\sigma \cdot(4 \pi)^{1 / 3}\left(3 V_{0}\right)^{2 / 3} \cdot \frac{2}{3} n^{*-1 / 3}= \\
& =4 \cdot \sigma \cdot V_{0}^{2 / 3} \cdot\left(\frac{\pi}{6}\right)^{1 / 3} \cdot n^{*-1 / 3}
\end{aligned}
$$

From Eq. (A6) it is easy to find the number of polymer molecules in a critical nucleus $\boldsymbol{n}$,

$$
n^{* 1 / 3}=\frac{4 \cdot \sigma \cdot V_{0}^{2 / 3} \cdot\left(\frac{\pi}{6}\right)^{1 / 3}}{k T \cdot \ln (\zeta)}
$$

Equation (A7) can be readily transformed to the form of (Eq. S2) by cubing and replacing the volume $\boldsymbol{V}_{\boldsymbol{0}}$ with the molar volume $\boldsymbol{V}_{\boldsymbol{M}}$ and the Boltzmann constant $\boldsymbol{k}$ with the gas constant $\boldsymbol{R}_{\text {gas }}$. 


\section{REFERENCES AND NOTES}

(1) Ruckenstein, E.; Djikaev, Y. S. Adv. Colloid Interfac. 2005, 118, 51.

(2) Kashchiev, D. Nucleation: Basic Theory with Applications; Butterworth-Heinemann: Oxford, 2000, p. 74.

(3) Zen, A.; Saphiannikova, M.; Neher, D.; Grenzer, J.; Grigorian, S.; Pietsch, U.; Asawapirom, U.; Janietz, S.; Scherf, U.; Lieberwirth, I.; Wegner, G. Macromolecules 2006, 39, 2162.

(4) Osawa, S.; Ito, M.; Iwase, S.; Yajima, H.; Endo, R.; Tanaka, K. Polymer 1992, 33, 914.

(5) Yu, F. Q. J. Chem. Phys. 2005, 122. 